\title{
Fusion of macrophages on lens implants resulting in the formation of giant cells *
}

\author{
J. Reimer Wolter \\ Departments of Ophthalmology and Pathology, University of Michigan Medical Center, Ann Arbor, MI 48109, USA
}

\begin{abstract}
Giant cells on lens implants are understood to be a sign of chronic irritation. These multinucleated cells develop from macrophages by fusion and they disappear in successful cases along with the free-moving macrophages, when the fixed fibroblast-like cells and the proteinaceous capsule have succeeded in creating a continuous and effective separation on the surface of a lens implant. Details about the stages in the development of giant cells on lens implants as well as their significance as typical representatives of chronic granulomatous inflammation are discussed.
\end{abstract}

\section{Introduction}

Routine use of the lens implant cytology technique [5] for the examination of all lens implants removed during surgery and from eyes obtained at enucleation or autopsy has opened the door to a new world of cell life, with a surprising amount of novelty and variation - even for expert cytologists. The cell formation presented in Fig. 1, for example, was shown to several experienced cytologists and pathologists with wide experience in inflammatory diseases. Their reaction was one of disbelief and excited surprise. There were guesses, suspicions and speculations, but no one had seen a cellular formation like this before, and no one was able to give it a name or explain its nature. This means that we have reached a frontier of present knowledge and that we have to collect and record our observations step by step and try to explain them on the basis of presently accepted cytologial knowledge. The purpose of the present paper is to add important details and some early generalizations to the initial descriptions and discussions concerning the giant cells on the surface of intraocular lens implants $[6,7,9-11,13,14,16]$.

\section{Histological description}

\section{Frist case}

Approximately 2.5 years after its implantation, an iris-supported polymethylmethacrylate lens implant was removed by Dr. Roger F. Meyer of this Eye Department during a keratoplasty for chronic corneal edema, from the eye of a 90 -year-old female. The pathological evaluation revealed

\footnotetext{
* Supported by The Research to Prevent Blindness, Inc. New York, USA
}

in the corneal region a fibrous pannus, stromal edema, scarring, neovascularization, and infiltration with polymorphonuclear and mononuclear cells. Descemet's membrane was thick and somewhat irregular. The endothelium showed diffuse loss of cells and pigment deposition in its protoplasm. The lens implant exhibited a continuous membrane on its surface. This was composed of a thin eosinophilic capsule with inactive-looking fibroblast-like cells and giant cells of different sizes. Hyperplastic vitreous strands were attached to some of the haptics which had been located in the back of the iris.

One strange cellular formation found on the front surface of the optic portion of this implant is of interest for the present paper (Fig. 1). This was located within the filmlike proteinaceous capsule, which also contained bipolar and star-shaped fibroblast-like cells as well as various giant cells in a rather irregular arrangement. The formation was large - about the size of a typical giant cell - oval, and flatly attached to the implant. Its substance stained distinctly eosinophilic and it contained about 20 cellular nuclei. Some of the nuclei were surrounded by a clear halo, while others were less distinct and piled on top of each other. The nuclei resembled those of active macrophages. The substance of the sharply limited unit resembled cellular protoplasm and this was fused in the center. All the nuclei were arranged in a ring around the periphery of the large protoplasmic formation. Furthermore, this had in one area a clearly recognizable fibroblast-like cell on its surface. In the region of another such cell on its surface there was an additional loose connection to an isolated fibroblast-like cell by way of one of its protoplasmic processes. It is important to emphasize that the cells in this protoplasmic unit were clearly recognizable, but they did not have distinct cell membranes.

\section{Second case}

Another medallion type of intraocular lens made of polymethylmethacrylate was implanted after intracapsular cataract extraction in 1978. Bullous keratopathy developed in 1981 and this was treated by Dr. Meyer with a successful penetrating keratoplasty. However, trauma on 25 January 1983 caused wound dehiscence with a prolapse of ocular content. During the repair of this rupture, Dr. Meyer removed the intraocular lens from the eye of the 72-year-old female - about 5 years after its implantation. The implant was fixed in $10 \%$ formalin, and again the léns implant cyto- 


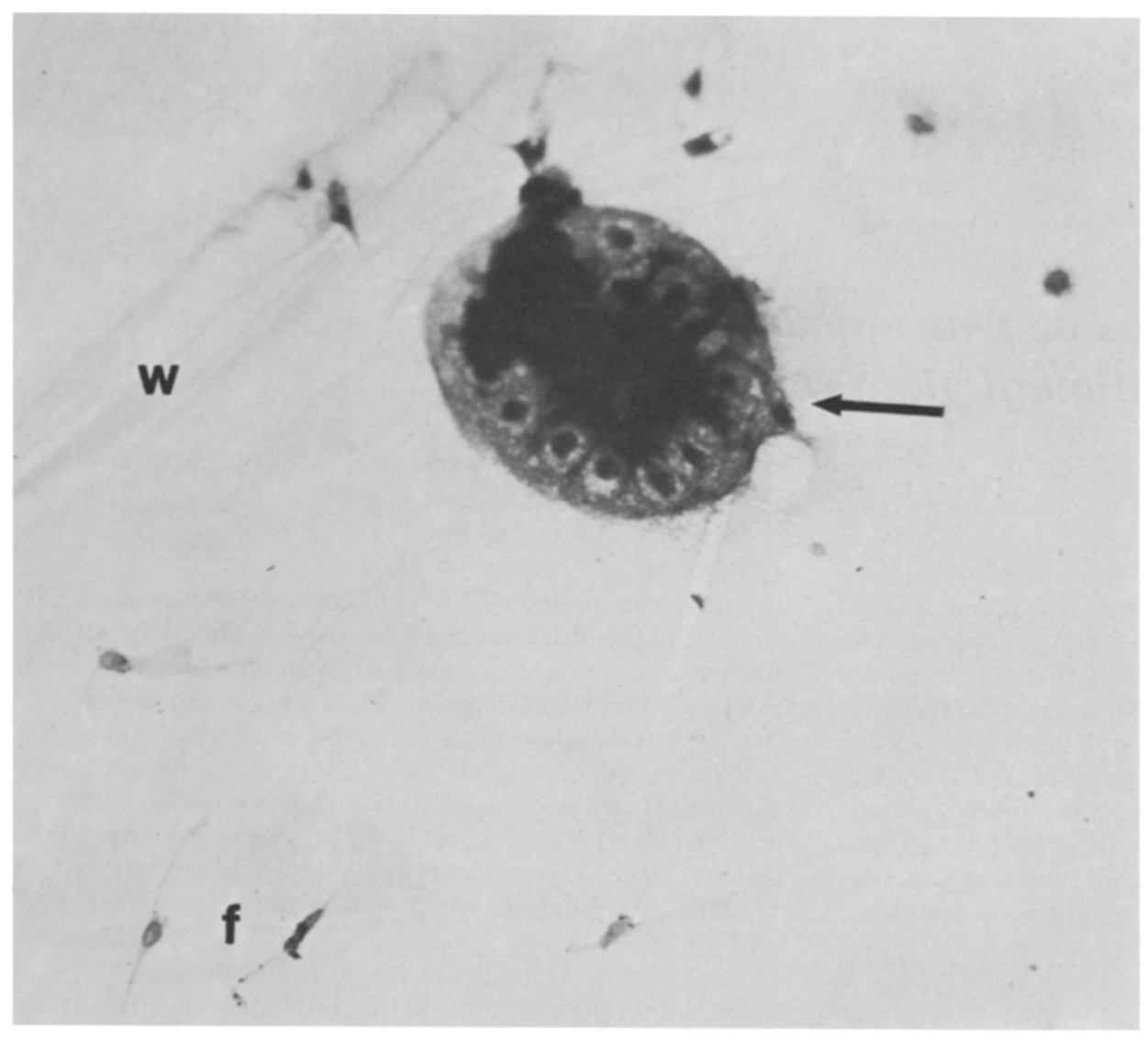

Fig. 1. First case, macrophages with light-staining protoplasm in a ringlike arrangement around a darkstaining central protoplasmic pool in an early stage of giant cell formation. The protoplasmic unit has relations to fibroblast-like cells on the surface of the implant (arrow). More fibroblast-like cells $(f)$ are seen evenly distributed all over the thin eosinophilic film on the surface of the implant. Artificial wrinkles in the film (w) on the upper left. Lens implant cytology technique, $\mathrm{H}$ and $\mathrm{E}$ stain, photomicrograph $\times 150$ logy technique was used to stain the cellular membrane on this implant with $\mathrm{H}$ and $\mathrm{E}$. The prolapsed tissues were processed separately and turned out to be iris with exudate and evidence of subacute nongranulomatous inflammation.

The implant was covered with a film of an acellular eosinonophilic substance. This contained polymorphonuclear leukocytes in addition to the macrophages, fibroblastlike cells, and giant cells usually seen on the surface of lens implants. The object of interest for the present study is a very unusual protoplasmic unit, the size of a large giant cell, which was found on this implant (Fig. 2). This contained in its protoplasm about 30 macrophage-like cells, but these did not have a separating cell membrane. The protoplasm of these cells again appeared lightly eosinophilic, like a halo around each nucleus within the more darkly eosinophilic substance of the unit. The nuclei were quite regular and round, and resembled those of free-moving macrophages.

\section{Third case}

A Choyce anterior chamber lens was placed after intracapsular cataract extraction in the right eye of this 69-year-old male in September 1981. The eye never had good vision and developed retinal detachment in May 1982. This was operated upon several times without success. All vision was lost and the eye became painful and chronically irritated. I enucleated the eye on 4 April 1983 and this was immediately fixed in $10 \%$ formalin. Gross study of the eye revealed subluxation of the anterior chamber implant, total retinal detachment, and much intraocular bleeding. Histological examination of the eye revealed diffuse nongranulomatous unvitis, vitreous hemorrhage, and a final stage of retinal detachment with evidence of extensive scleral surgery.

Cytological study of the implant showed large portions of a proteinaceous membrane containing fibroblast-like cells and great numbers of giant cells of many different types and sizes. Some of the largest giant cells had central accumulations of pale-staining nuclei (Fig. 3) and a sharply outlined protoplasmic border. The peripheral protoplasm often had a somewhat light and foamy appearance. However, there were also some cells with a very dense and dark central accumulation of virtually confluent nuclei (Fig. 3). On this implant it was common to see free-moving macrophages and fibroblast like cells extending partly or completely on to the protoplasm of giant cells (Fig. 3).

\section{Experiment}

A polymethylmethacrylate lens implant was placed into the peritoneal space of a mouse under sterile conditions. When the implant was removed after 5 days, a continuous membrane of macrophages was seen to cover its surface continuously [15]. Large numbers of giant cells were found on the surface of the plastic implant in addition to the macrophages.

Careful study of the giant cells on the surface of this implant allowed for the observation of all stages in the development of the large giant cells: from loose accumulations of macrophages over several stages of protoplasmic fusion to the formation of complete and typical giant cells. Figure 4 is one view of the surface of the implant with piles of closely associated macrophages ( $p$ in Fig. 4). The first stage of giant cell formation (1 in Fig. 4) consists in 

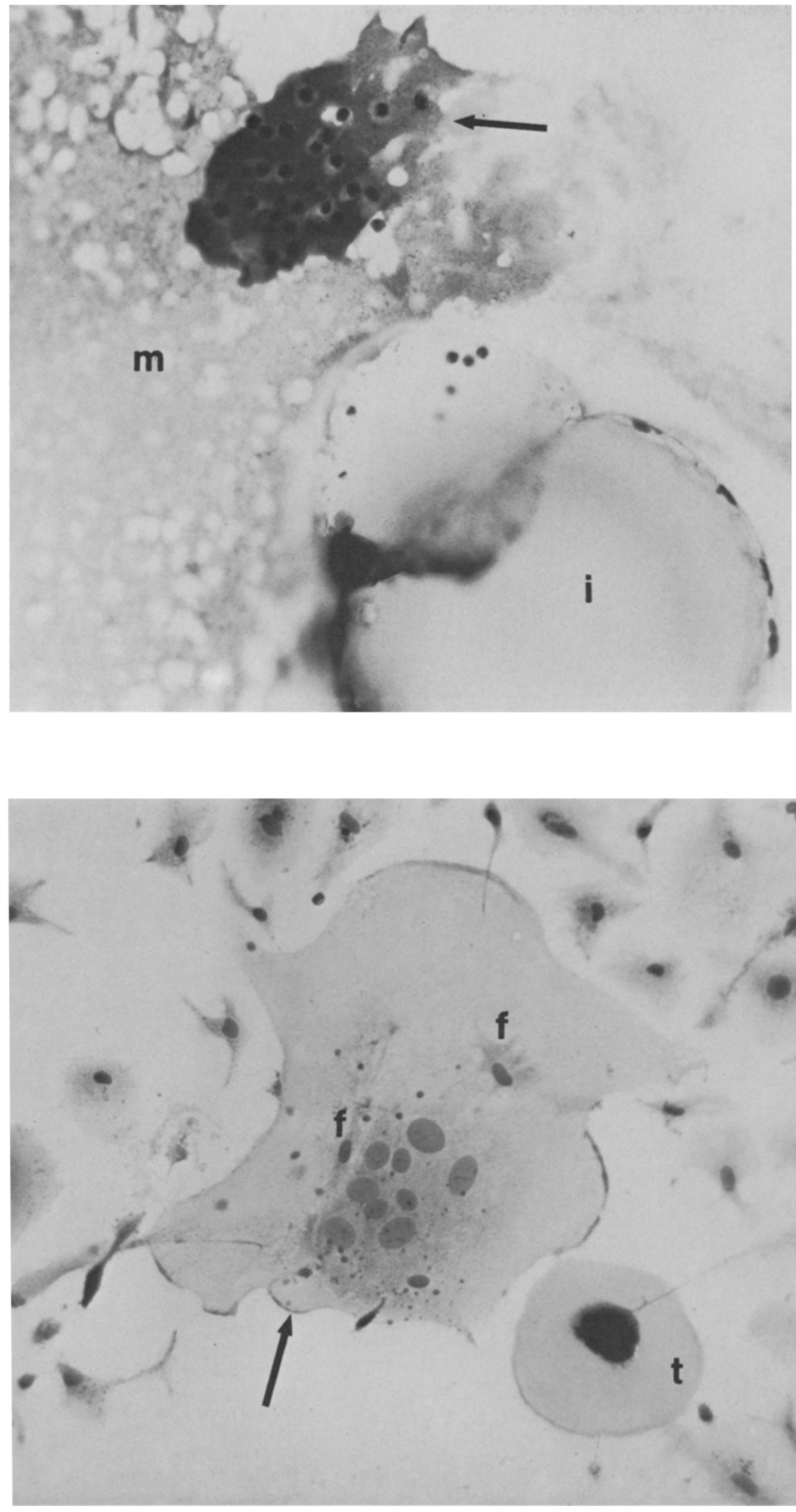

Fig. 2. Second case, protoplasmic unit containing 33 macrophages with preserved halo-like cells bodies (arrow) on the surface of the lens implant. A ring of fibroblast-like cells are found around the insertion of a haptic $(i)$. Portions of an irregular eosinophilic membrane $(m)$ are seen on the plastic surface. Lens implant cytology technique, $\mathrm{H}$ and $\mathrm{E}$ stain, photomicrograph $\times 150$
Fig. 3. Third case, one well-developed and sharply limited giant cell (arrow) on the lens implant. A second targetlike giant cell $(t)$ has a very dense accumulation of nuclei in the center. Fibroblast-like cells are surrounding the giant cells and they are also seen on top of the large giant cell $(f)$. Lens implant cytology technique, $\mathrm{H}$ and $\mathrm{E}$ stain, photomicrograph $\times 150$ 

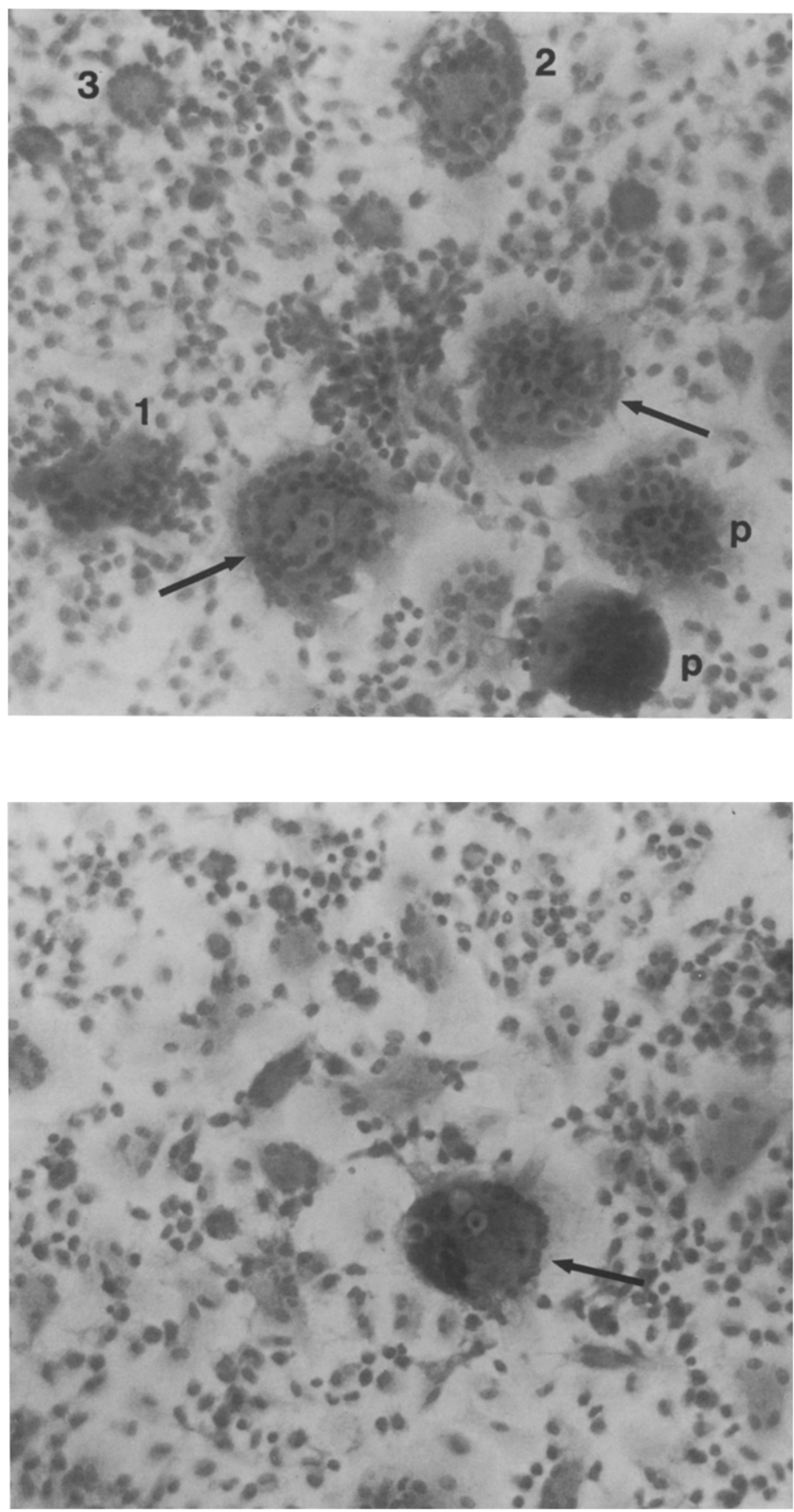

Fig. 4. Macrophages covering surface of lens implant after 5 days in peritoneal space of a mouse. Some of the macrophages have formed pilelike accumulations of cells $(p)$. In the first stage of giant cell formation a pool of common protoplasm develops in the center (1). In the second stage the macrophages start a ring-like arrangement and the pool enlarges (2). In the third stage (3) a Langhans type of cell has developed. Lens implant cytology technique, $\mathrm{H}$ and $\mathrm{E}$ stain, photomicrograph $\times 150$
Fig. 5. Macrophages on lens implant after 5 days in the mouse peritoneal space. Giant cell in second stage of its formation (arrow) containing macrophages with preserved halo-like protoplasm. One of these cells is seen at the border of the protoplasmic unit. Lens implant cytology technique, $\mathrm{H}$ and $\mathrm{E}$ stain, photomicrograph $\times 150$ 


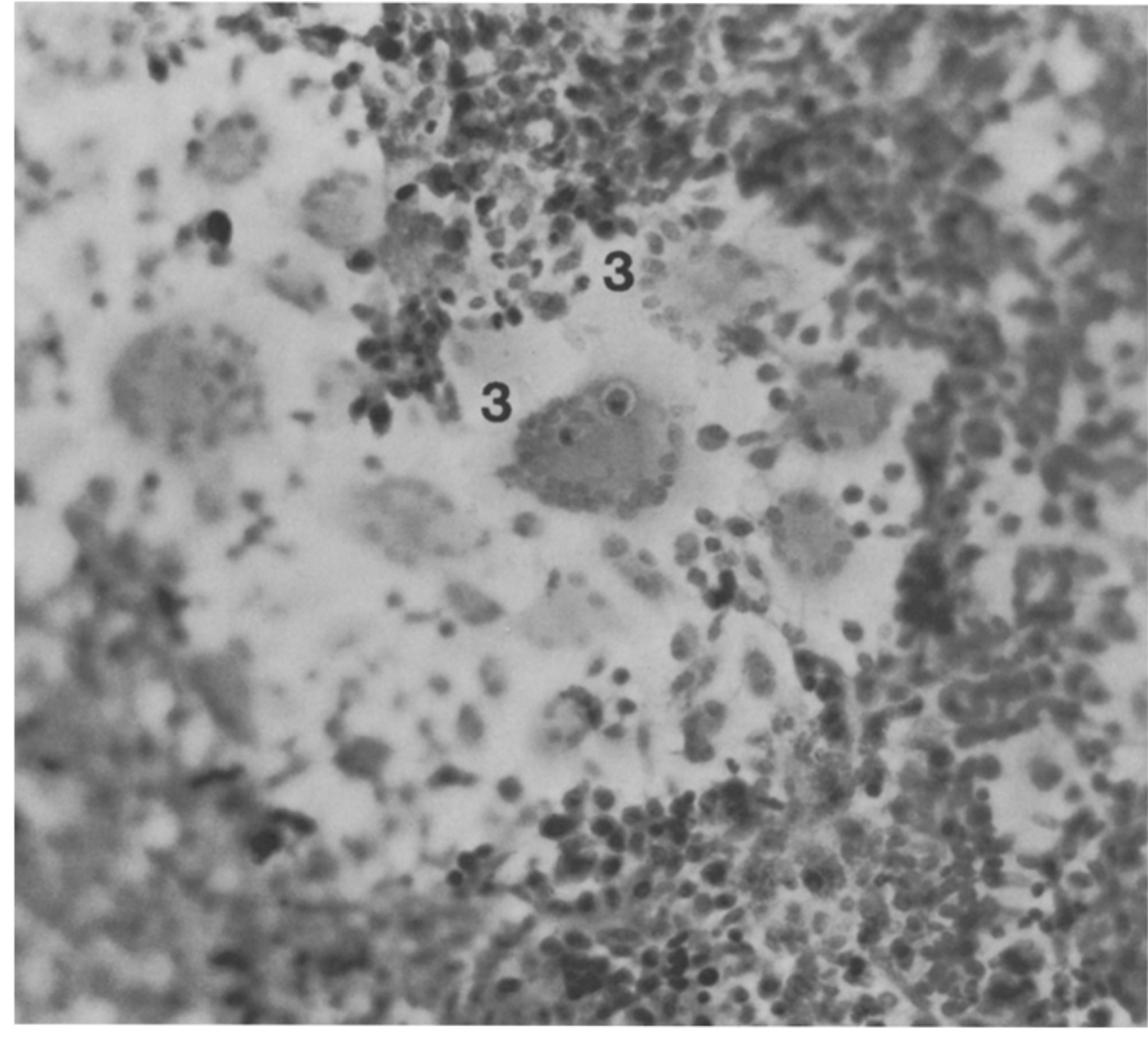

Fig. 6. Macrophages and giant cells on lens implant after 5 days in peritoneal space of a mouse. Giant cells in the third stage have the appearance of Langhans cells (3). One additional macrophage with halo-like protoplasm is clearly seen in one of the giant cells. Lens implant cytology technique, $\mathrm{H}$ and $\mathrm{E}$ stain, photomicrograph $\times 150$ protoplasmatic fusion in the center of these piles, resulting in a homogeneous protoplasmatic center ( 1 in Fig. 4). In the next stage the central protoplasm enlarges and the nuclei around assume a somewhat irregular order (2 in Fig. 4). With further progress in the development of the giant cells the nuclei form a more perfect single or double ring around the increasing central pool of protoplasm ( 3 in Fig. 4). It is most important to emphasize that no mitoses are seen anywhere in the piles of macrophages. Furthermore, it is remarkable to see some macrophages with a lighter halolike protoplasm within the piles of cells (arrows in Fig. 4). In some instances these macrophages were seen at the margin of the giant cell unit - either leaving or entering this (arrow in Fig. 5). The fully developed giant cells had an extensive pool of protoplasm in the center and the nuclei were arranged in a single or double ring around the midperiphery of the protoplasm ( 3 in Fig. 6). It is important to emphazise that one stage in the formation of the giant cells on the implant in the abdomen of the mouse was a rather dark-staining protoplasmic unit containing macrophages with a halo-like light-staining protoplasm (see arrow in Fig. 5).

\section{Discussion}

Most textbooks state that giant cells can develop either by fusion of the protoplasm of active macrophages or by repeated divisions of cellular nuclei of macrophages without cell division. The present study shows that giant cells on intraocular lens implants develop by fusion of macrophages. Under these conditions, I have not as yet seen any mitoses or other signs of cell division in macrophages or giant cells - even on implants with numerous cells under the most active circumstances in clinical or experimental situations. The question as to whether or not giant cells on lens implants can ever develop by nuclear division has to remain open.

The protoplasmic units containing macrophages seen on the surface of the lens implants in the first two cases are believed to be newly discovered interim stages between an accumulation of macrophages and the formation of true giant cells on the surface of lens implants. The macrophages have joined to a unit and created a pool of common protoplasm. However, the protoplasm of the single cells forming this pool is still clearly visible due to the lighter staining. In the first case the macrophages have an orderly arrangement around the border of the unit. In the second case the cells are of irregular arrangement in the pool of common protoplasm. It is very probable that the unit in the first case is slightly more advanced in its development to a giant cell than that of the second case. The view that macrophages can join, form a common pool of protoplasm and thus produce giant cells, is supported by the observations of more stages of the same process in the experimental situation with peritoneal mouse macrophages. It is remarkable to observe that macrophages can clearly enter or leave the protoplasmatic units at this early stage of giant cell formation from or into the surrounding surface area of the implants. Evidence for this fact has accumulated in many instances with human and mouse macrophages on implants. The example of the third case is used to show a typical giant cell on a lens implant in a human eye. Furthermore, the strange related giant cells with a target-like 
central arrangement of one large or several small nuclei is of interest because of its unusual appearance. Their nature and purpose are unknown. It is also important to point out that macrophages often extend onto the surface of giant cells on lens implants - in spite of the fact that the macrophages otherwise have a tendency to form a monolayer.

Giant cells in general are differentiated by the arrangement of their nuclei in relation to the protoplasm. They are known as the Langhans type when their nuclei are all arranged around their periphery. Foreign-body giant cells, in contrast, have their nuclei rather evenly distributed throughout their protoplasm. Finally, Touton giant cells have a central or paracentral accumulation of nuclei and contain peripheral protoplasmatic vacuoles usually filled with lipids. Giant cells on or next to lens implants may resemble all three of these types. In addition there are the giant cells with a very dense clump of nuclei in the center of their protoplasm seen on lens implants or with one giant central nucleus, described in a recent paper [10].

In contrast to the common feeling that giant cells are an expression of very chronic inflammatory processes, Warren [4] has recently shown convincingly that "giant cells are exceedingly short-lived and will disappear rapidly if a supply of new macrophages is cut off." This can be observed after whole body radiation for example. Warren [4] has also shown that giant cells have " poor phagocytic abilities." The study of giant cells on lens implants has certainly supported these two observations. The giant cells disappear from lens implants when the ocular situation stabilizes, and when the macrophages on the surface of an implant become inactive. The giant cells do not take part in the very impressive phagocytic activities of the sessile macrophages (fibroblast-like cells). However, the giant cells often do contain pigment granules [8] or large vacuoles with an empty appearance. It has become difficult to believe in the significance of the different types of giant cells. Warren feels that the foreign-body type of giant cell is a precursor of the Langhans type. I certainly have seen both types mixed on the same lens implant. Most numerous on lens implants are giant cells that resemble Touton cells and have a ring of nuclei in the midperiphery, vacuoles in the peripheral protoplasm, and additional small vacuoles in the center. The fact that both Spector [3] and Epstein [1] have postulated that giant cells function as a mechanism to delete unwanted and unnecessary cells from granulomatous inflammations may indicate that giant cells are much less important in this process than we tend to think.

In the modern diagnosis of granulomatous inflammations, the classification of the giant cells has become less important. More attention is paid to the arrangement of all the cells involved in the granulomatous reaction as well as to the demonstration of its cause. In the region of the eye giant cells are common in diseases caused by autoimmune hypersensitivity or fungus infection, for example. Sympathetic and phacoanaphylactic endophthalmitis, as well as ocular involvement in rheumatoid arthritis or hypersensitivity reaction against degenerating Descemet's membrane, are good examples $[2,17]$. However, the same giant cells in principle may be seen in a chalazion, surrounding foreign substance in the inner eye or even on a retained and hardened lens nucleus [12].

The present experimental studies with a lens implant in the peritoneal space of the mouse show many stages of giant cells being formed by macrophages accumulating in a pile and by undergoing protoplasmic fusion in the center. It is not only possible to clearly recognize some of the macrophages as cell units within the pooled protoplasm, but the impression is that additional macrophages can enter the giant cells. They either give up their protoplasmic entity and persist only as a nucleus - or remain a cell within the pool of protoplasm that may again leave the protoplasmic unit of the giant cells. The cells that undergo protoplasmic fusion to form the giant cell start out in disorder - and this stage resembles what is known as a foreignbody giant cell. With time the macrophages in the unit take the orderly ring-like or U-shaped arrangement typical of Langhans giant cells. The presence of giant cells on lens implants with abundant newly arrived and free-moving macrophages is considered in principle to be an indication of unrest and irritation. Giant cells commonly occur next to the insertion of haptics, especially when these are made of Supramid or Prolene. Giant cells are usually massive on luxated or otherwise grossly disturbed implants. Welltolerated implants in contrast are covered with an even proteinaceous film containing inactive fibroblast-like cells, but these typically exhibit no, or only very few, giant cells.

All giant cells are closely related to macrophages, of course, and they are an active part of the systemic phagocyte system. They are also relatives of the Kupffer cells of the liver, the alveolar macrophages of the lung, and the fixed and free macrophages of spleen, lymphnodes, and bone marrow. This type of cell is not a normal inhabitant of the eye. Implantation of intraocular lenses is a situation where macrophages and other cells derived from macrophages take permanent residence in the eye - and as yet we do not understand all of the consequences. Macrophages are in general very powerful cells. They can create lysing enzymes against bacteria and have been shown to attach to neoplastic cells and kill them in vitro. It is disturbing to realize that the common combination of giant cells and activated macrophages on the surface of lens implants, with some lymphocytic infiltration in the adjacent structures of iris and ciliary body, allows for the pathological calssification of the whole situation as at least a nonimmunologic kind of smoldering granulomatous endophthalmitis.

\section{References}

1. Epstein WL (1977) Granuloma formation in man. Pathobiol Annu 7:1-30

2. Green WR, Zimmerman LE (1967) Granulomatous reaction to Descemet's membrane. Am J Ophthalmol 64:555-559

3. Spector WG (1976) Immunologic components of granuloma formation. Epithelioid cells, giant cells, and sarcoidosis. Ann NY Acad Sci 278:3-6

4. Warren KS (1980) The cell biology of granulomas (aggregates of inflammatory cells) with a note on giant cells. In: Weissman $G$ (ed) Handbook of inflammation II. Elsevier/North Holland, Amsterdam-New York-Oxford, pp 543-557

5. Wolter JR (1982) Lens implant cytology. Opthalmic Surg 13:939-942

6. Wolter JR (1982) Foreign body giant cells on intraocular lens implants. Graefe's Arch Clin Exp Ophthalmol 219:103-111

7. Wolter JR (1982) Cell life on the surface of lens implants. Graefe's Arch Clin Exp Opthalmol 218:244-249

8. Wolter JR (1982) Pigment in cellular membranes on intraocular lens implants. Opthalmic Surg 13:726-732

9. Wolter JR (1983) Reactive membrane on lens implant: three months after implantation. Graefe's Arch Clin Exp Ophthalmol $220: 53-57$ 
10. Wolter JR (1983) Morphology of the capsule-like portion of the reactive membranes on intraocular lens implants. Graefe's Arch Clin Exp Ophthalmol 220:58-65

11. Wolter JR, Felt DP (1983) Proliferation of fibroblast-like cells on failing intraocular lenses. Ophthalmic Surg 14:57-64

12. Wolter JR (1983) Foreign body reaction to firm nuclear substance. Opthalmic Surg 14:135-138

13. Wolter JR (1983) Pseudophaco-anaphylactic endophthalmitis? Graefe's Arch Clin Exp Opthalmol 220:160-166

14. Wolter JR (1983) Foreign body giant cells selectively covering haptics of intraocular lens implants: indicators of poor toleration? Ophthalmic Surg (in press)
15. Wolter JR, Kunkel SL (1983) Abdominal implantation of intraoculae lenses: resulting in the formation of reactive membranes. Graefe's Arch Clin Exp Ophthalmol (in press)

16. Wolter JR, Lichter PR (1983) Fibroblast-like cells on intraocular lens implants: phagocytizing erythrocytes. $\mathrm{Br} \mathbf{J}$ Ophthalmol (in press)

17. Wolter JR, Johnson FD, Meyer RF, Watters JA (1971) Acquired autosensitivity to degenerating Descemet's membrane in a case with anterior uveitis in the other eye. Am J Ophthalmol 72:782-786

Received May 13, 1983 